\title{
DEFORMATIONS OF NORMAL VECTOR FIELDS AND THE GENERALIZED MINKOWSKI PROBLEM
}

\author{
BY HERMAN GLUCK ${ }^{1}$
}

Communicated by M. H. Protter, June 7, 1971

In this note I announce a solution to the

Generalized Minkowski problem. Find an embedding of the $m$-sphere $S^{m}$ into Euclidean space $R^{m+1}$, whose Gaussian curvature is preassigned as a continuous, strictly positive function on $S^{m}$.

Such embeddings are shown to exist without exception for $m \geqq 2$, but with certain necessary exceptions for $m=1$, the result in this case standing as a converse to the classical Four Vertex Theorem for plane curves.

While the above problem lies in the realm of differential geometry in the large, its solution comes via differential topology by studying deformations of normal vector fields on a closed smooth manifold $M^{m}$ in $R^{m+1}$.

Detailed proofs may be found in [9], while a leisurely exposition of the converse to the Four Vertex Theorem appears in [10]. I am indebted to Eugenio Calabi, Jerry Kazdan and Frank Warner for many helpful conversations, and particularly to Warner for introducing me to the problem in the first place and for pointing out its relation to the study of normal vector fields on spheres.

1. The generalized Minkowski problem. In the classical Minkowski problem, one starts only with those continuous strictly positive curvature functions $K: S^{m} \rightarrow R^{1}$ which satisfy the integrability condition $\int_{S^{m}} N(p) / K(p) d \Omega=0$, where $N(p)$ is the unit outward normal vector to the unit $m$-sphere $S^{m}$ in $R^{m+1}$. In return, these preassigned curvatures are realized not by arbitrary embeddings of $S^{m}$ into $R^{m+1}$, but by inverses of Gauss maps, and one also gets a corresponding uniqueness theorem up to parallel translations. The reader can find an historical survey of this problem in [9]; the original sources are Minkowski [13], [14], Bonnesen and Fenchel [6], Lewy [12],

AMS 1970 subject classifications. Primary 53C45, 53C20, 57D25; Secondary 57D50.

Key words and phrases. Minkowski problem, Four Vertex Theorem, Gaussian curvature, Riemannian metric, convex surface, convex hypersurface, vector field, normal vector field, deformation.

1 The author acknowledges partial support from the National Science Foundation grant GP-19693. 
Alexandrov [1], [2], [3] Pogorelov [17] and Nirenberg [16].

In the generalized Minkowski problem, all continuous strictly positive functions $K: S^{m} \rightarrow R^{1}$ are considered, and we seek to realize these preassigned curvatures by unrestricted embeddings of $S^{m}$ into $R^{m+1}$. There is no corresponding uniqueness result.

The generalized Minkowski problem in $R^{3}$ would be immediately solved by an affirmative answer to the following question posed by Nirenberg: Given a smooth positive function $K: S^{2} \rightarrow R^{1}$, does there exist a closed convex surface $M^{2} \subset R^{3}$ and a conformal homeomorphism $G: S^{2}$ $\rightarrow M^{2}$, such that the Gaussian curvature of $M^{2}$ at the point $G(p)$ is $K(p)$ for all $p \in S^{2}$ ? The attacks on Nirenberg's question have all been via variational methods for partial differential equations, without success. Recently Kazdan and Warner [11] have discovered smooth strictly positive functions on $S^{2}$ which cannot be the Gaussian curvature of any Riemannian metric on $S^{2}$ conformal to the standard one. In spite of this negative answer to Nirenberg's question for $S^{2}$, the corresponding conjecture for other closed surfaces (with conformal embedding into $R^{3}$ replaced by existence of a Riemannian metric conformal to a standard one) has met with some success. Results for closed surfaces of genus $\geqq 2$ have been obtained by Berger [4], for the torus and Klein bottle by Kazdan and Warner [11], and for the projective plane by Moser [15].

The solution to the generalized Minkowski problem for embeddings of $S^{m}$ into $R^{m+1}$ with preassigned Gaussian curvature is separated, for convenience, into three cases: $m=1, m=2$ and $m \geqq 2$.

TheOREM I. Let $k: S^{1} \rightarrow R^{1}$ be a continuous strictly positive function which is either constant or else has at least two maxima and two minima. Then there exists an embedding $G: S^{1} \rightarrow R^{2}$ of $S^{1}$ onto a convex simple closed curve $M^{1}$, such that the curvature of $M^{1}$ at $G(\phi)$ is $k(\phi)$ for all $\phi \in S^{1}$. Furthermore, if $k$ is of class $C^{r}$, then $G$ is of class $C^{r+1}$ and $M^{1}$, if reparametrized by arc length, is of class $C^{r+2}$ for all $r \geqq 0$.

Maxima and minima are counted in the strict sense. That is, a constant function has no maxima and minima, and a nonconstant function $k: S^{1} \rightarrow R^{1}$ has one maximum and one minimum if there are points $p_{1}$ and $p_{2}$ on $S^{1}$ such that $k$ is a nondecreasing function along each of the two arcs of $S^{1}$ from $p_{1}$ to $p_{2}$. Otherwise $k$ has at least two maxima and two minima.

According to the classical Four Vertex Theorem [5, p. 30], the conditions of Theorem I are necessary as well as sufficient, so that Theorem I may be regarded as a converse to the Four Vertex Theorem. 
THEOREM II. Let $K: S^{2} \rightarrow R^{1}$ be a continuous strictly positive function. Then there exists an embedding $G: S^{2} \rightarrow R^{3}$ of $S^{2}$ onto a closed convex surface $M^{2}$ whose Gaussian curvature at the point $G(p)$ is $K(p)$ for all $p \in S^{2}$. Furthermore, if $K$ is of class $C^{r}$ then $G$ is of class $C^{r+1}$ for $r=0$ or for $r \geqq 2$.

THEOREM III. Let $K: S^{m} \rightarrow R^{1}$ be a continuous strictly positive function, $m \geqq 2$. Then there exists a $C^{\infty}$ diffeomorphism $h: S^{m} \rightarrow S^{m}$, diffeotopic to the identity, and a closed convex hypersurface $M^{m} \subset R^{m+1}$ with Gauss "map" ${ }^{2}: M^{m} \rightarrow S^{m}$, such that for each Borel subset $V \subset S^{m}$,

$$
\left|\gamma^{-1} V\right|=\int_{\nabla} \frac{1}{K h^{-1}(p)} d \Omega,
$$

where $\left|\gamma^{-1} V\right|$ is the surface area of $\gamma^{-1} V$ on $M^{m}$.

If, under the above circumstances, $\gamma: M^{m} \rightarrow S^{m}$ is a homeomorphism, then $G=\gamma^{-1} h: S^{m} \rightarrow M^{m} \subset R^{m+1}$ is an embedding such that the Gaussian curvature of $M^{m}$ at the point $G(p)$ is $K(p)$ for all $p \in S^{m}$.

Although the language is different, the content of Theorem III is substantially that of Theorem II, with two exceptions: we do not know whether the Gauss "map" $\gamma: M^{m} \rightarrow S^{m}$ is a homeomorphism, and even if so, we cannot predict the smoothness of $G$ from that of $K$. It follows from [7], however, that if $\gamma$ is a homeomorphism then $M^{m}$ is smooth of class $C^{\mathbf{1}}$.

2. Deformations of normal vector fields. Let $M^{m}$ be a connected closed smooth (say $C^{\infty}$ ) manifold in $R^{m+1}$, and for each point $p \in M$, let $N(p)$ denote the unit outward normal vector to $M$ at $p$. Let $f: M \rightarrow R^{1}$ be a continuous function, not necessarily positive. Then the vector field $f(p) N(p), p \in M$, will be called a (continuous) normal vector field on $M$. Let $h: M \rightarrow M$ be a $C^{\infty}$ diffeomorphism, diffeotopic to the identity. At the same time that $h$ slides a point $p$ of $M$ over to its image $h(p)$, we may imagine the normal vector $f(p) N(p)$ to $M$ at $p$ being dragged along with $p$, its length remaining fixed, until it becomes the normal vector $f(p) N(h p)$ to $M$ at $h(p)$. Writing $f(p) N(h p)$ $=f h^{-1}(h p) N(h p)$, we see that $h$ moves the normal vector field $f(p) N(p)$ to the normal vector field $g(p) N(p)$, where $g=f h^{-1}$. Under these circumstances, we say that the two normal vector fields on $M$ are deformations of one another.

The integral of a given normal vector field $f(p) N(p)$ on $M$ is the vector $\int_{M} f(p) N(p) d A$ in $R^{m+1}$, which is generally nonzero, and we wish to deform the vector field so as to make its integral over $M$ vanish.

${ }^{2} \mathrm{~A}$ "map" is a possibly many-valued map. 
THEOREM A. If $M^{m} \subset R^{m+1}, m \geqq 2$, is a closed smooth manifold in Euclidean space, then any continuous normal vector field on $M$ can be deformed so that afterwards its integral over $M$ vanishes.

There are some exceptions to this theorem if $m=1$, that is, if $M^{1}$ is a smooth simple closed curve in the plane. A convex curve $M^{1} \subset R^{2}$ is strictly convex if it contains no nondegenerate line segments; otherwise it is weakly convex.

THEOREM B. If $M^{1} \subset R^{2}$ is a smooth simple closed curve in the plane, then any continuous normal vector field $f(p) N(p)$ on $M^{1}$ can be deformed so that afterwards its integral over $M^{1}$ vanishes, with two exceptions:

(1) If $f$ has just one maximum and one minimum and $M^{1}$ is strictly convex, then the required deformation never exists.

(2) If $f$ has just one maximum and one minimum and $M^{1}$ is weakly convex, then the required deformation exists if and only if there is a nondegenerate arc on $M^{1}$ along which the value of $f$ is constant but not extremal.

Theorem I follows by using Theorem $\mathrm{B}$ to find a diffeomorphism $h: S^{1} \rightarrow S^{1}$ such that $\int_{S^{1}}\left(1 / k h^{-1}(\phi)\right) N(\phi) d \phi=0$, and then appealing to standard results in elementary differential geometry. Theorems II and III follow by using Theorem $\mathrm{A}$ to find a diffeomorphism $h: S^{m} \rightarrow S^{m}$ such that $\int_{S^{m}}\left(1 / K h^{-1}(p)\right) N(p) d \Omega=0$, and then appealing to the solution of the classical Minkowski problem.

These transition arguments are all trivial, so the real problem is to prove Theorems $\mathrm{A}$ and $\mathrm{B}$.

3. The idea behind the proofs of Theorems A and B. The proofs of Theorems A and B are fairly long, but the idea behind them is easily described by analogy with the usual topological proof of the Fundamental Theorem of Algebra [8, pp. 306-307]. Given a continuous normal vector field $f(p) N(p)$ on $M^{m} \subset R^{m+1}, m \geqq 2$, we must hunt for a diffeomorphism $h: M \rightarrow M$, diffeotopic to the identity, such that $\int_{M} f h^{-1}(p) N(p) d A=0$. This is an equation with unknown $h$ contained in Diff $\left(M^{m}\right)$, the group of diffeomorphisms of $M$ with the $C^{\infty}$ topology. The map $I: \operatorname{Diff}\left(M^{m}\right) \rightarrow R^{m+1}$,

$$
I(h)=\int_{M} f h^{-1}(p) N(p) d A
$$

is continuous and is the analogue of a complex polynomial function.

During the proof we construct a certain $(2 m+2)$-cell $B^{2 m+2}$ in $\operatorname{Diff}\left(M^{m}\right)$. The cell $B^{2 m+2}$ is the analogue of the complex plane, and within it a root of $I$ will be found. Inside $B^{2 m+2}$ we construct a certain 
$m$-sphere $\Sigma^{m}$, which is the analogue of a circle of large radius in the complex plane.

We then show that $I\left(\Sigma^{m}\right)$ misses the origin in $R^{m+1}$, and that $I \mid \Sigma^{m}: \Sigma^{m} \rightarrow R^{m+1}-\{0\}$ is an essential map. Since $\Sigma^{m}$ is contractible within $B^{2 m+2}$, we are immediately assured of the existence of a root (indeed, infinitely many roots) of the equation $I(h)=0$, just as in the proof of the Fundamental Theorem of Algebra. Since $B^{2 m+2}$ was constructed to contain the identity $1_{M}, h$ is diffeotopic to $1_{M}$ and Theorem A follows.

Most of the proof of Theorem A also handles the positive part of Theorem $\mathrm{B}$, but the negative aspects of Theorem $\mathrm{B}$ require individual arguments.

\section{BIBLIOGRAPHY}

1. A. D. Alexandrov, Zur Theorie gemischter Volumina konvexer Körper. I, II, III, Mat. Sb. 2 (1937), 947-972; 2 (1937), 1205-1238; 3 (1938). 167.

2. - Von der Flächenfunktion eines konvexen Körpers, Mat. Sb. 6 (48) (1939),

3. - Smoothness of the convex surface of bounded Gaussian curvature. C. R. (Dokl.) Acad. Sci. URSS 36 (1942), 195-199. MR 4, 69.

4. M. Berger, On Riemannian structures of prescribed Gauss curvature for compact two-dimensional manifolds, J. Diff. Geometry 5 (1971) (to appear).

5. W. Blaschke, Vorlesungen über differential Geometrie, Berlin, 1930; reprint, Chelsea, New York, 1967.

6. T. Bonnesen and W. Fenchel, Theorie der konvexen Körper, Julius Springer, Berlin, 1934.

7. H. Busemann, Convex surfaces, Interscience Tracts in Pure and Appl. Math., no. 6, Interscience, New York, 1958. MR 21 \#3900.

8. S. Eilenberg and N. Steenrod, Foundations of algebraictopology, Princeton Univ. Press, Princeton, N. J., 1952. MR 14, 398.

9. $\mathrm{H}$. Gluck, The generalized Minkowski problem in differential geometry in the large, Ann. of Math. (to appear).

10. - The converse to the Four Vertex Theorem, l'Enseignement Math. (to appear).

11. J. Kazdan and $\mathrm{F}$. Warner, Integrability conditions for $\Delta u=k-K e^{\alpha u}$ with applications to Riemannian geometry, Bull. Amer. Math. Soc. 77 (1971), 819-823.

12. $\mathrm{H}$. Lewy, On the existence of a closed surface realizing a given Riemannian metric, Proc. Nat. Acad. Sci. U.S.A. 24 (1938), 104-106.

13. H. Minkowski, Allgemeine Lehrsätze über konvexer Polyeder, Nachr. Ges. Wiss. Göttingen 1897, 198-219.

14. - Volumen und Oberfläche, Math. Ann. 57 (1903), 447-495.

15. J. Moser (to appear).

16. L. Nirenberg, The Weyl and Minkowski problems in differential geometry in the large, Comm. Pure Appl. Math. 6 (1953), 337-394. MR 15, 347.

17. A. V. Pogorelov, Regularity of a convex surface with given Gaussian curvature, Mat. Sb. 31 (73) (1952), 88-103. (Russian) MR 14, 679.

University of Pennsylvania, Philadelphia, Pennsylvania 19104 